\title{
Radiological Diagnosis and Approach: Glomus Tumour at Finger Tip
}

\section{Taori $\mathbf{A}^{1}$, Sakhi $\mathbf{P}^{1}$, Malukani $\mathbf{K}^{2}$, Patel $\mathbf{K}^{1}$}

${ }^{1}$ Department of Radiodiagnosis, ${ }^{2}$ Department of Pathology, Sri Aurobindo Medical College and PG Institute, Indore, Madhya Pradesh, India

\begin{abstract}
A Glomus tumor is a rare benign neoplasm arising from the glomus body which is a neuroarterial structure and mainly found under the nail, on the fingertip or in the foot. We present here a case of a 60 Year old female with a long history of pain in her left Index finger. She underwent radiological investigations of Skiagram Hand, High resolution ultrasonography with color Doppler of Local Region (Finger), and MRI Finger; thereby diagnosis of glomus tumor was made. Subsequently it was surgically removed; Histopathological result was consistent with glomus tumour.
\end{abstract}

Keywords: Finger Tip, Glomus Tumour, MRI, USG

\section{Introduction}

A glomus tumor (also known as a solitary glomustumor, solid glomus tumor, or glomangioma) is a rare benign neoplasm arising from the glomus body and mainly found under the nail, on the fingertip or in the foot. They account for less than $2 \%$ of all soft tissue tumors. ${ }^{1}$ Glomus tumor was first described by Hoyer in 1877, while the first complete clinical description was given by Masson in $1924 .^{2}$

The exact incidence of glomus tumor is unknown. Glomus tumors are usually

Correspondence to: Dr. Abhijeet Taori, Department of Radiodiagnosis, Sri Aurobindo Medical College and PG Institute, Indore, Madhya Pradesh, India. Email: abhijittaori@gmail.com solitary and small lesions. The multiple variant is rare, accounting for less than $10 \%$ of all cases. The vast majority are found in the distal extremities, particularly in the hand, wrist, foot, and under the fingernails. Solitary glomus tumors, particularly subungual lesions, are more common in females than in males. Multiple lesions are slightly more common in males. The average age at presentation is from 30 to 50 years of age, although can occur at any age. ${ }^{3}$ The probable misdiagnosis of many of these lesions as hemangiomas or venous malformations also makes an accurate assessment of incidence difficult.

\section{Case}

A 60 year old Indian middle aged female presented to the neurology OPD with 3 Year history of pain at the tip of left Index Finger. 
She had numerous visits to different health care specialist in the past. There were no systemic complaints.

She had "small seed" like mass lesion at the tip of the digit. The patient complained of sharp pain whenever pressure was applied to the volar tip of her index finger during day to day activity.

The physical examination revealed no discoloration of the complained digit or other digits and the nail along with nail bed appeared normal. A distinct circumscribed mass could not be well appreciated by the neurologist but a seed like small lesion could be just felt at tip of index finger of left hand, patient had severe pain on palpating the central area of the pulp. Routine laboratory investigations were within normal limits. Thereafter patient was referred to Department of Radiodiagnosis for radiological Investigations.
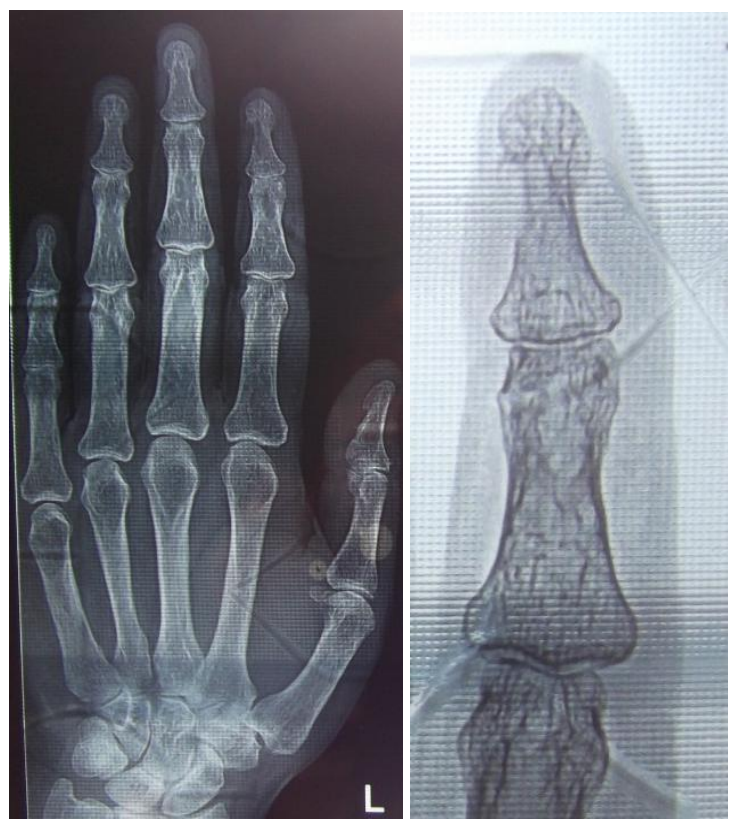

Fig. 1: Skiagram Hand. Conspicuous bone erosion of the first distal phalanx in Left Index Finger.
The Skiagram of Hand showed distinctly visible bone erosion at the first distal phalanx in Left Index Finger (Fig.1).

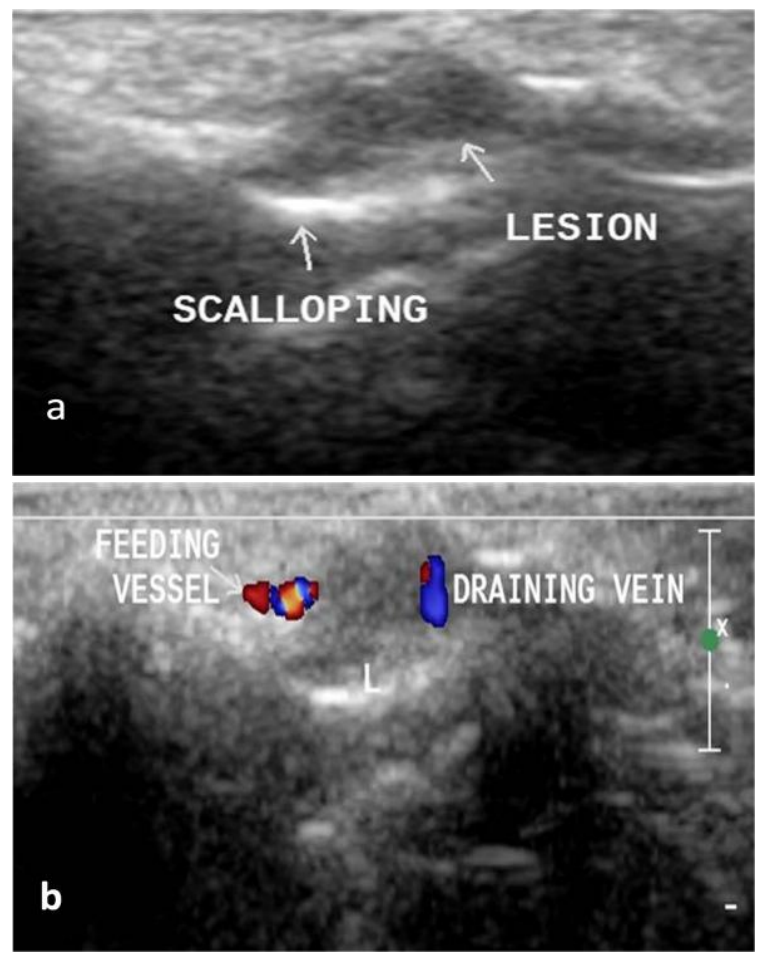

Fig. 2 a\&b: High resolution USG with color Doppler of Local Region (Finger). High resolution ultrasonography with color Doppler of Local Region reveals a focal, solid, hypoechoic mass beneath the nail of index finger, with conspicuous bone erosion of the first distal phalanx and vascularity within the tumor.

USG of Finger showed a focal, solid, hypoechoic mass beneath the nail of left index finger measuring approximately $5.5 \times 3.1 \mathrm{~mm}$, with conspicuous bone erosion of the first distal phalanx and vascularity within the tumor (Fig. 2a \& b). The MRI obtained showed an oval post gadolinium enhancing oval circumscribed lesion at the tip of distal phalanx of index finger of left hand (Fig.3).

On basis of Radiological diagnosis surgical excision of the tumour was planned. A volar approach to the index finger was made, and 

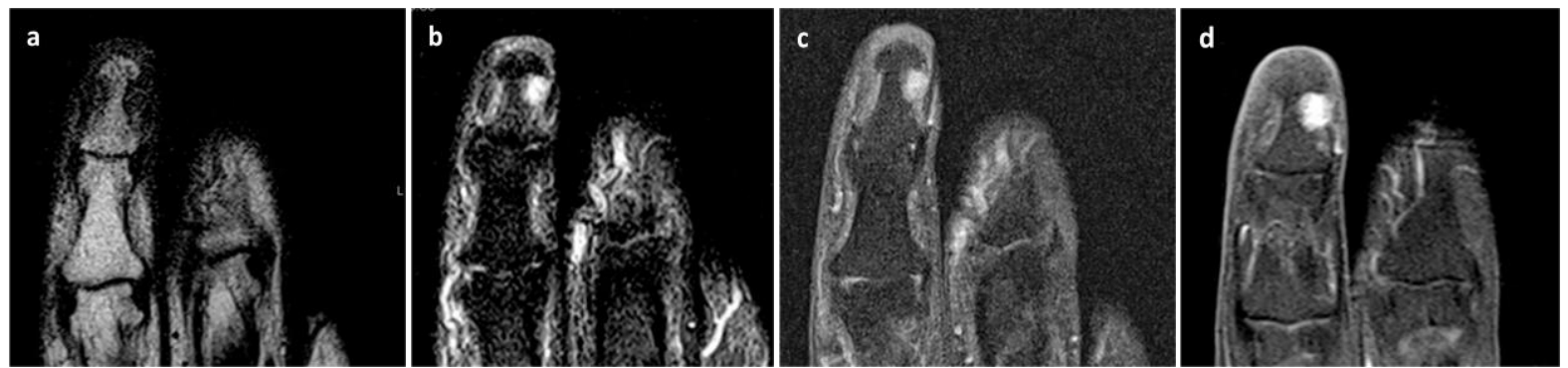

Fig.3 a (T1) b (T1 TIRM) c (PD Fat Sat) d (T1+C): MRI Finger. The MRI revealed an oval mass at the tip of distal phalanx of index finger of left hand. The lesion appears as hypointense ill-defined oval lesion on T1 weighted images and hyperintense on T1 TIRM and PD FatSat Images.It enhances avidly on contrast on T1 post-gadolinium images.

excision was performed. A circumscribed oval lesion measuring $5 \times 3 \mathrm{~mm}$ was identified, without gross surrounding tissue abnormality. The resulting mass was sent for histopathology. It revealed sheet of numerous monotonous round glomus cells with peripheral eosinophilic cytoplasm and round to oval nuclei. These findings were consistent with the pre-operative diagnosis of glomus tumor (Fig.4).

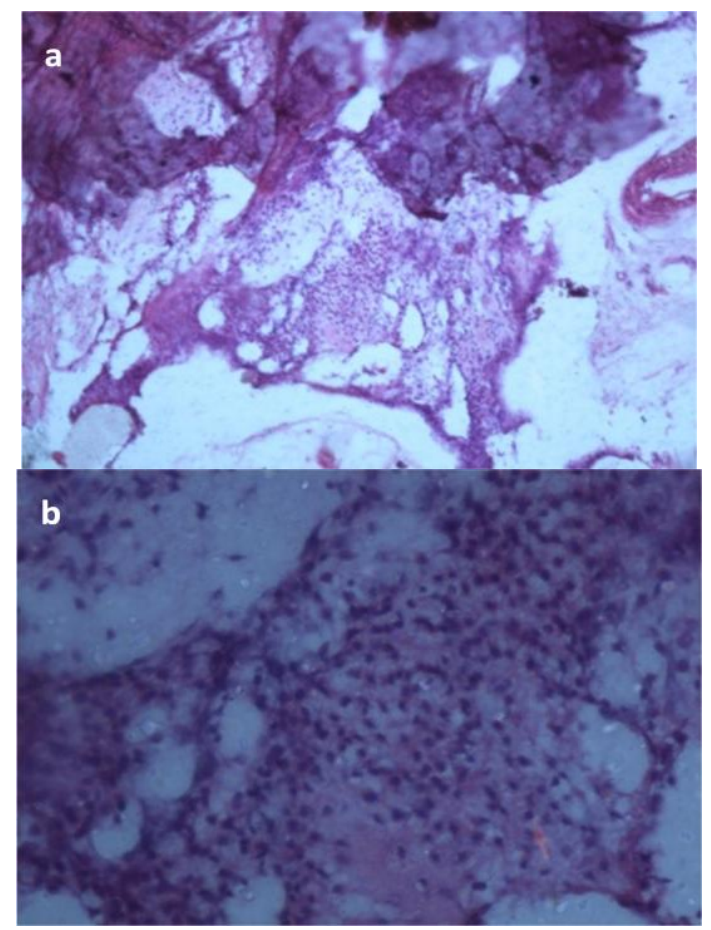

Fig.4:Histopathology. a. Photomicrograph of glomus tumour with peripheral bone tissue (H \& E 100X) b. Photomicrograph showing sheet of numerous monotonous round glomus cells with peripheral eosinophilic cytoplasm and round to oval nuclei (H \& E 400X).

On follow up, the patient reported complete relief of her pre-operative symptoms.

\section{Discussion}

A glomus tumor is a rare benign neoplasm arising from the glomus body and mainly found under the nail, on the fingertip or in the foot accounting for less than $2 \%$ of all soft tissue tumors. ${ }^{1}$

This benign condition has an unusually high morbidity to the patient before the correct diagnosis is made. The patient with glomus tumor seeks medical attention early, but the mass is frequently too small to be identified on physical examination. ${ }^{4}$ Although the classic triad of moderate pain, temperature sensitivity, and point tenderness has been described, these are nonspecific and not all may be present. ${ }^{5}$ Furthermore as the mass is usually very small in diameter, it is very difficult to palpate.

Plain radiographs are minimally helpful and are typically normal until the bony erosion 
occurs at the later stages of the disease. Thus it is difficult to correctly diagnose this lesion initially. Clinical history and carefully performed physical examination significantly narrow the differential diagnosis.

Ultrasound has been advocated to aid in the diagnosis of glomus tumor, it is operator and techniques dependent. ${ }^{6}$ Magnetic Resonance is the imaging modality of choice when evaluating soft tissue masses.

Glomus tumor is a vascular entity, reflecting it's typically dark on T1 and bright MRI appearance on T2 weighted images. Postgadolinium and fat saturation images can further help in delineating the mass. This signal pattern can be seen with any vascular tumor but the location at the digits and its small size should lead one to suspect glomus tumor in most cases.

Surgery is the treatment of choice. There is only low rate of recurrence. ${ }^{7}$

\section{References}

1. International Agency for Research on Cancer (2002). Pathology and Genetics of Tumors of Soft Tissue and Bone. St. Louis: WHO Press. Pp. 136-137.

2. Gombos, Z; Zhang, PJ (2008 Sep). "Glomus tumor". Archives of pathology \& laboratory medicine 132 (9): 1448-52.

3. Carroll RE, Berman AT. Glomus tumors of the hand. J Bone Joint Surg. 1972; 54A(4):691-703.

4. Mohler DG, Lim CK, Martin B. Glomus tumor of the plantar arch: A case report with magnetic resonance image findings. Foot \& Ankle Int. 1997; 18(10):672-674.

5. Rettig AC, Strickland JW. Glomus tumor of the digits. J Hand Surg. 1977; 2A(4):261-265.

6. Fornage BD. Glomus tumors in the fingers: Diagnosis with S. Radiology. 1988; 167(1):183-185.

7. Kim DH. Glomus Tumor of the Finger Tip and MRI Appearance. Iowa Orthop J. 1999; 19:136-8. 